\title{
Dynamic Strength of Iron at High Pressures and Strain Rates
}

\author{
Vatsa Gandhi®, Suraj Ravindran®, and Guruswami Ravichandran® \\ Division of Engineering and Applied Science, California Institute of Technology, Pasadena, California 91125, USA
}

(Received 9 July 2021; revised 25 October 2021; accepted 17 December 2021; published 7 January 2022)

\begin{abstract}
Accurate modeling of meteorite impacts, and deformation of planetary cores require characterization of the flow strength and in-elasticity of iron in its different phases. In this Letter, we investigate the flow strength of both the ambient $\alpha$ phase and high-pressure $\epsilon$ phase of iron at strain rates of $1 \times 10^{5} \mathrm{~s}^{-1}$ and pressures up to $42 \mathrm{GPa}$ using high-pressure-pressure shear plate impact experiments. We report the strength of the $\epsilon$ iron to be significantly higher than $\alpha$ phase but consequently one order smaller than the previously reported dynamic strength at high pressures. The complete stress-strain response of the $\epsilon$ phase is reported for the first time.
\end{abstract}

DOI: 10.1103/PhysRevLett.128.015705

Iron has many geological and planetary implications as it is the primary constituent in the inner core of outer planets such as Uranus and Neptune [1], rocky exoplanets, and the inner rocky planets including Earth where core pressures exceed $330 \mathrm{GPa}$ [2]. Understanding the dynamic inelastic behavior of iron at high pressures is critical for many applications. These include modeling the physics of hypervelocity impacts of iron-rich meteorites [3], the formation of craters on Earth and moon [4], and planetary impact such as the giant-impact hypothesis on the formation of the Earth-moon system [5-7]. Plasticity behavior of iron is also important for describing deformation mechanisms of the inner cores of rocky planets [8], for example due to seismic waves generated from earthquakes. However, modeling iron at these high pressures is difficult because it undergoes a martensitic phase transformation [9] from the bodycentered-cubic (bcc) $\alpha$ phase to hexagonal-closed-pack (hcp) $\epsilon$ phase $[10,11]$ when subjected to $13 \mathrm{GPa}$ of pressure.

Martensitic phase transformations provide a unique opportunity to study strength behavior as they play a crucial role in enhanced material properties and expand the material design space for various applications. An illustrative and important example of this is iron alloys. Martensitic steels formed by rapid quenching yield high strength, high fatigue-resistant materials [12], whereas transformation induced plastic (TRIP) steels partially transform to martensite upon deformation thus resulting in improved strength and ductility [13]. This further motivates the study of plasticity behavior of $\epsilon$-phase iron as it can help novel materials for load bearing applications.

While the shocked equation of state $[7,14]$, the picosecond to nanosecond range of characteristic transition times [15], and even the importance of shear on the initiation of the bcc-hcp transformation [16] have provided considerable insight regarding the material behavior of the $\epsilon$ phase, the strength and plasticity behavior of this martensite under dynamic conditions is still not fully explored. Iron completely reverts to its $\alpha$ phase upon release of pressure hence this unstable nature of $\epsilon$ iron has severely limited the possibility of developing a material model to describe its inelastic behavior.

Diamond anvil compression (DAC) experiments coupled with XRD have provided initial insight regarding the low strain-rate strength of iron at core pressures. Hemley et al. [17] observed pressure hardening of $\epsilon$ iron and deduced a shear stress of $\tau \approx 10 \mathrm{GPa}$ at 200-300 GPa of pressure. This significantly differs from more recent DAC experiments $[8,18]$ which suggest half the strength and indicate a linear relationship between shear strength and pressure. While the static data is promising, the limitations include the determination of only a single data point for strength measurements at a constant pressure and the indirect nature of XRD yields high uncertainties.

Despite the importance, dynamic strength characterization of $\epsilon$ iron has been limited. Extended x-ray absorption fine structure (EXAFS) measurements on laser shocked polycrystalline iron by Ping et al. [19] provide preliminary, and indirect dynamic strength characterizations on $\epsilon$ iron under quasi-isentropic conditions. The authors interpolate plastic work contribution to the temperature measurements to estimate the strength of $\epsilon$ iron up to $560 \mathrm{GPa}$ of pressure. However, this measurement involves very high uncertainties on the order of $50 \%$. A more recent study by Huntington et al. [20] utilizes Rayleigh-Taylor instability experiments to observe the ripple growth of iron, laser shocked to $100 \mathrm{GPa}$, to extract the material strength. The authors obtained yield strengths of $40 \mathrm{GPa}$ at these pressures which are comparable to Ping et al. [19], but are on the cusp of theoretical strength limits.

In this Letter, we report the measurement of the pressure dependent dynamic strength behavior of $\alpha$ and $\epsilon$ iron under quasi-isentropic loading conditions, using the recently developed sandwiched high-pressure-pressure shear plate 
impact [21] (HP-PSPI) technique. Experiments are conducted on iron at pressures ranging from 10 to $42 \mathrm{GPa}$ and a constant compressive and shearing strain rate of $10^{5} \mathrm{~s}^{-1}$. Additionally, simulations are conducted to model the equation of state and plasticity of both phases of iron. By varying parameters within the plasticity model, the simulated normal and transverse free surface velocities are matched with experimentally determined quantities to determine the first ever complete shear stress-strain behavior of iron.

In PSPI experiments [22], the oblique impact generates both longitudinal and shear waves in the front anvil, which first compresses and then shears the sample at a constant pressure. The HP-PSPI experiments were performed on annealed Armco iron (purity 99.8\%) with an average grain size $70 \mu \mathrm{m}$ using a slotted barrel powder gun. A $150 \mu \mathrm{m}$ thin iron sample was sandwiched between tungsten carbide (WC) anvils to ensure the sample equilibrates to a constant pressure before it is sheared. The sandwich is assembled by placing the iron sample between the anvils such that the iron-WC interfaces are directly in contact with one another and friction between the materials transmits the shear wave. Both normal and transverse free surface velocity measurements [23] were conducted using photon doppler velocimetry (PDV) [24]. Figure 1(a) illustrates the experimental setup and a detailed description can be found in Sec. I of the Supplemental Material (SM) [25].

Because of plasticity within the anvil material above its Hugoniot elastic limit (HEL), conventional elastic analysis of extracting strength from transverse velocity profiles is not feasible. For this reason, we conduct finite element simulations via ABAQUS/Explicit [31] and match simulated

(a)
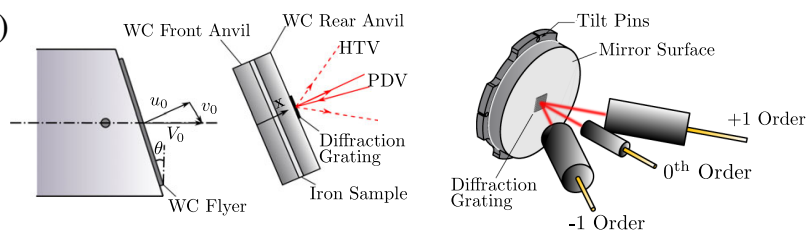

(b)

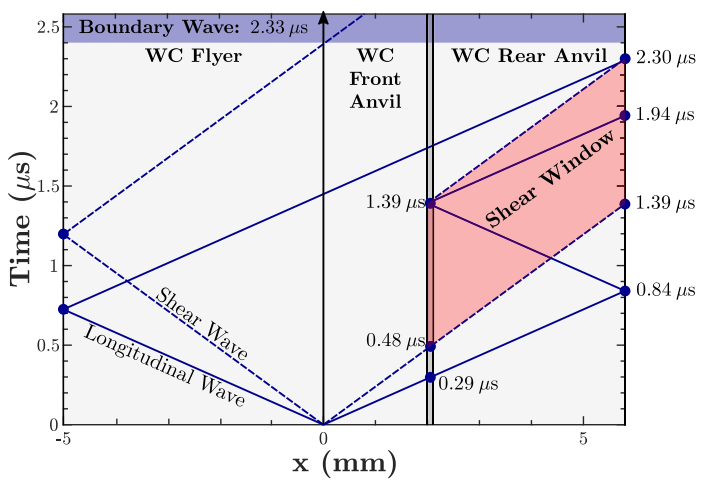

FIG. 1. (a) Schematic of the pressure shear plate impact setup in sandwich configuration. (b) Distance-time $(x-t)$ diagram for tungsten carbide-iron sandwich assuming elastic wave propagation. velocities with the experimental measurements [21,32] to extract a material model for the iron sample. In particular, the peak transverse velocity is directly related to the strength and flow behavior of iron and thus we determine simulation parameters to match peak velocities of the experimental data. This sandwich configuration and plastically deforming anvil analysis methodology has been successfully validated using various anvil materials on copper [32]. Thus, the key results in this Letter represent the physics of iron and are not from an unknown error in the experimental or analysis method. For a known tungsten carbide material model [32], the only unknowns required to match our experimental velocity profiles are equation of state and strength parameters for iron. For $\alpha$ iron, a Johnson-Cook strain and strain-rate hardening model with temperature softening from Sadjadpour et al. [33] was implemented. For $\epsilon$ iron, we employ the JohnsonCook hardening law with temperature dependence and a Steinberg-Cochran-Guinan (SCG) [34] pressure dependence on yield strength as follows:

$$
\begin{aligned}
Y= & \left(Y_{0}+B \epsilon^{n}\right)\left(1-T^{* m}\right) \\
& \times\left[1+\left(\frac{Y_{p}^{\prime}}{Y_{0}}\right) \frac{P}{\eta^{1 / 3}}-\left(\frac{G_{T}^{\prime}}{G_{0}}\right)(T-300 \mathrm{~K})\right]
\end{aligned}
$$

where $G_{0}=83 \mathrm{GPa}$ [8] is the shear modulus at ambient conditions, $T^{*}=\left[\left(T-T_{r}\right) /\left(T_{m}-T_{r}\right)\right]$ with $T$ corresponding to the temperature, $T_{r}=300 \mathrm{~K}$ the reference temperature, $T_{m}=1811 \mathrm{~K} \mathrm{[33]}$ the melting temperature, and $\eta=$ $\left(\rho / \rho_{0}\right)$ the compression ratio with $\rho$ being the density, and $\rho_{0}=7870 \mathrm{~kg} / \mathrm{m}^{3}$ [33] the reference density. From Eq. (1), the initial yield $Y_{0}$, the strain hardening terms, $B$ and $n$, and the temperature softening exponent, $m$ were varied in simulations to match experimental velocities whereas the other parameters were obtained from previous literature. The strain-rate dependence in the Johnson-Cook model was neglected as all experiments were conducted at a nominally constant strain rate, $10^{5} \mathrm{~s}^{-1}$. Within our simulations, the temperature contributions include both plastic work from high strain-rate deformations (assuming Taylor-Quinney factor of $\beta=1[33,35])$ and shock heating due to high pressure jumps which was modeled using an equation of state (EOS). The EOS of iron for all experiments was modeled using a well calibrated Helmholtz potential by Boettger and Wallace [14] in conjunction with a continuum timedependent phase transformation numerical scheme by Hayes [36] and Andrews [37]. This was implemented to accurately capture the two-phase region, specifically for the $15.8 \mathrm{GPa}$ experiment. Details of the EOS and the numerical scheme can be found in Secs. II and III of the SM, respectively.

When considering the yield strength scaling with respect to pressure, the SCG model approximates a one-toone relationship between the pressure dependence behavior of the shear modulus and yield strength $\left[\left(1 / Y_{0}\right)(\partial Y / \partial P)=\right.$ $\left.\left(1 / G_{0}\right)(\partial G / \partial P)\right]$. However, recent experiments on tantalum [38] and copper [32] have shown the yield strength 
scaling to be 2 and 2.6 times that of shear modulus, respectively. This was based on the decrease of dislocation mobility at higher pressures and the shear modulus scaling of work required to move the dislocations [39]. In the present study, a one-to-one scaling of shear modulus and yield strength with respect to pressure was sufficient to simultaneously match both normal and transverse velocities for $\epsilon$ iron. We adapt the $\epsilon$-iron shear modulus data from static experiments [8]: $\left(1 / G_{0}\right)(\partial G / \partial P)=0.0246 \mathrm{GPa}^{-1}$ and $\left(1 / G_{0}\right)(\partial G / \partial T)=1.08 \mathrm{kK}^{-1}$. The plot of the shear modulus as a function of pressure can be found in Mao et al. [40]. This governs the chosen parameters for the pressure hardening and temperature softening law. The other parameters in the Johnson-Cook model were fit to match the free surface velocity profiles of the four experiments.

The parameters determined for the $\epsilon$-iron hardening model through a sensitivity study and linear least squares minimization problem (see SM) are below:

\begin{tabular}{cccccc}
$Y_{0}[\mathrm{GPa}]$ & $B[\mathrm{GPa}]$ & $n$ & $m$ & $\left(Y_{P}^{\prime} / Y_{0}\right)\left[\mathrm{GPa}^{-1}\right]\left(G_{T}^{\prime} / G_{0}\right)\left[\mathrm{kK}^{-1}\right]$ \\
\hline 2.0 & 2.4 & 0.73 & 1.08 & 0.0246 & 1.08 \\
\hline
\end{tabular}

The experimentally measured normal and transverse velocity profiles along with the simulated profiles at each corresponding pressure are displayed in Fig. 2. The experimental details can be found in the SM (Table V). Because of plasticity within our WC anvils and the thickness of our iron sample, no phase transformation wave is observed in the normal profile. Additionally, the very thin sample does not affect the normal profile in terms of the HEL and peak free-surface velocity. However, due to the impedance mismatch between iron and WC, pressure reverberations upon the arrival of the initial longitudinal wave slightly affect the rise times of the shock wave. This further validates the quasi-isentropic loading condition in the sandwiched sample.

Unlike the normal velocity profile, the transverse profiles primarily depict the strength behavior of iron. We note the imposed transverse velocities in these experiments ranged from $100-110 \mathrm{~m} / \mathrm{s}$ to ensure a shear strain rate on the order of $10^{5} \mathrm{~s}^{-1}$ within the iron sample. The measured transverse velocities are below the imposed velocity at the impactortarget interface indicating dispersion of the shear wave due to plasticity in the iron sample. As the longitudinal wave traverses through the sample, at high pressures, the material yields. Therefore, the shear wave traversing through this yielded material will disperse [21]. For anvil materials stronger than iron, e.g., tungsten carbide, the maximum dissipation of the shear wave occurs within the sandwiched sample. We employ the von Mises yield criterion where the yield condition for shear-normal coupling is $\frac{3}{4} s_{x x}^{2}+\tau_{x y}^{2}=$ $\frac{1}{3} Y^{2}$ [21] with $Y$ as the yield stress, $\tau_{x y}$ as the shear stress, and $s_{x x}$ as the normal component of the deviatoric stress tensor. Although the longitudinal wave is sufficient to yield
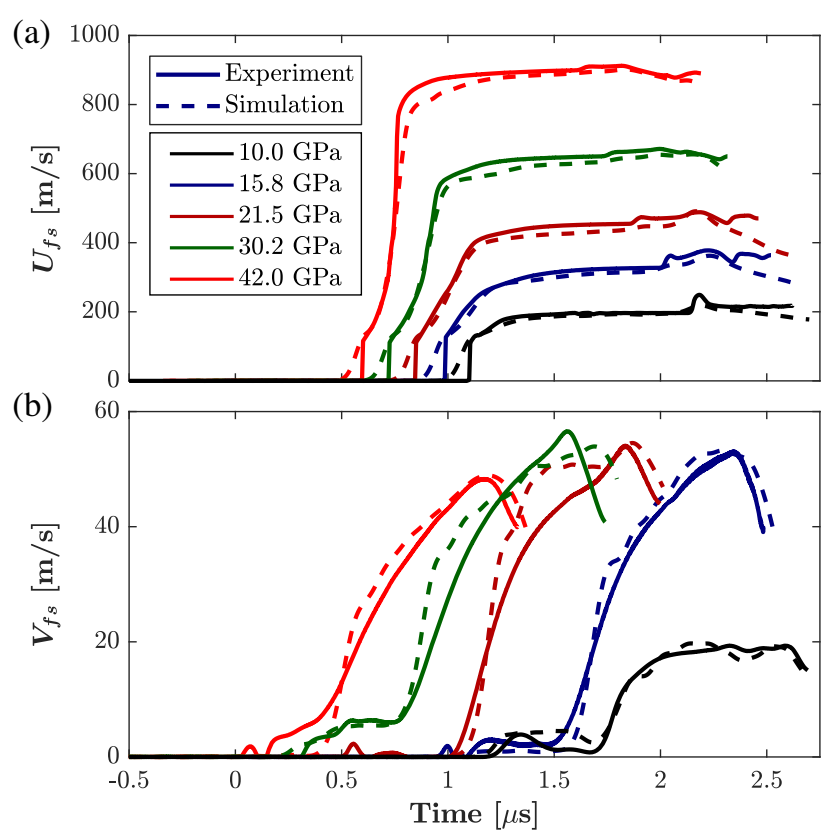

FIG. 2. (a) Normal and (b) transverse free surface velocity profiles at varying pressures for pure iron. The solid lines correspond to experimental records and the dashed curves correspond to simulated profiles. Both the normal and transverse velocity profiles (except for the $15 \mathrm{GPa}$ curves) have been shifted for clarity in visualizing the results.

the material, the arrival of the shear wave increases $\tau_{x y}$ while decreasing $s_{x x}$ to ensure the material stays on the yield surface. If the shear wave arrives at an amplitude of $\tau_{x y}=Y / \sqrt{3}$, the deviatoric stress vanishes and the shear stress provides a direct measurement of the yield behavior of the material. Therefore, the transverse velocity profiles correlate to the strength of the iron in its respective phase.

From initial inspection, we observe a significant increase in transverse velocity at $15.8 \mathrm{GPa}$ compared to $10 \mathrm{GPa}$. This indicates $\epsilon$ iron having a much higher strength than the ambient $\alpha$ iron. However, we cannot discern strength directly from the transverse velocity profile as the peak velocities at higher pressures are similar to the peak at 15.8 GPa. The primary reason for this phenomenon is due to increased plasticity in anvils at higher pressures resulting in larger shear wave dispersion at the rear anvil. Therefore, the transverse velocities themselves are insufficient to conclude strength behavior of iron at high pressures highlighting the importance of forward modeling to extract the stress-strain behavior. The dashed curves in Fig. 2 correspond to the simulated velocity profiles.

By constraining the shear strain rate at $\sim 10^{5} \mathrm{~s}^{-1}$ through varying impact angles, the complete pressure dependent strength and flow behavior of $\epsilon$ iron was extracted from the simulations for a full range of shear strains, Fig. 3(a). From our data we make two main conclusions, (1) the strength of iron in its $\epsilon$ phase is more than double that of the $\alpha$-phase, 


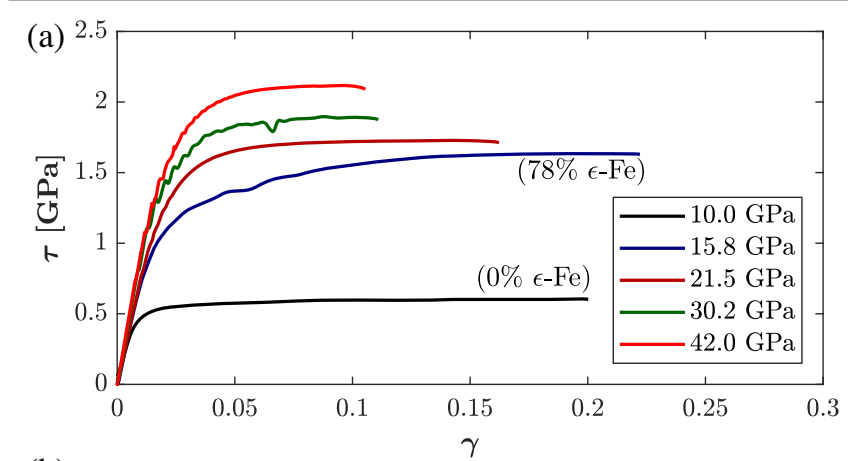

(b)

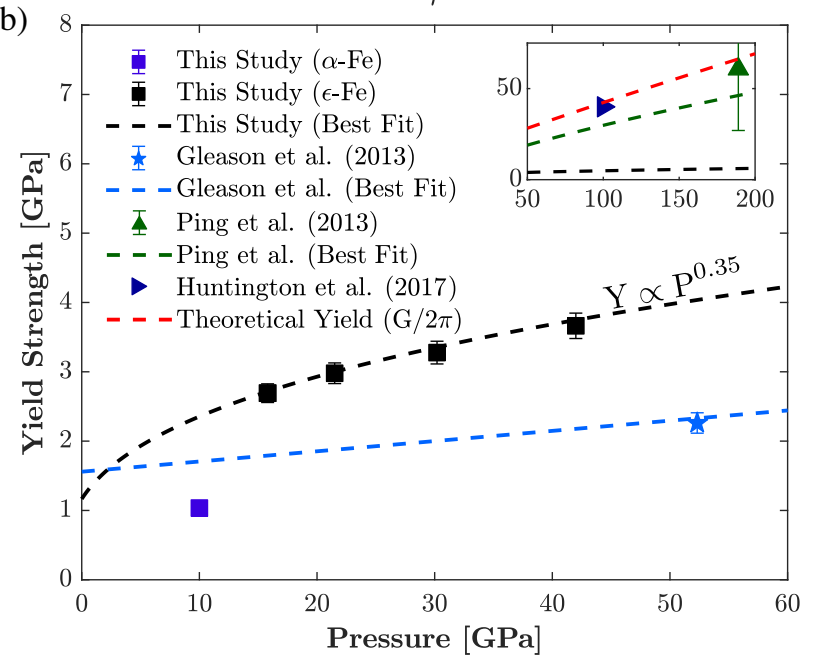

FIG. 3. (a) Shear stress vs shear strain of iron for varying pressures and (b) comparison of von Mises yield strength at $10 \%$ shear strain with data from literature. The best fit curve (black dashed line) follows a power law relation $\left(Y \propto P^{0.35}\right)$ compared to the linear relation obtained from static experiments by Gleason and Mao [8]. The main plot compares our data with other static experiments and the inset contains data from other dynamic experimental studies alongside our best fit curve. The dashed red curve corresponds to the theoretical yield strength $(G / 2 \pi)$ obtained from shear modulus data from [8].

and (2) the dynamic strength of $\epsilon$ iron is an order of magnitude lower than previously observed.

The yield strength $(Y=\sqrt{3} \tau)$ at $\gamma=0.1$ of iron at $15.8 \mathrm{GPa}$ of pressure ( $78 \% \epsilon$ phase based of the EOS model [14]) is $2.69 \pm 0.13 \mathrm{GPa}$. In contrast, at $10 \mathrm{GPa}$ of pressure, the yield strength of $\alpha$ iron is $1.04 \pm 0.05 \mathrm{GPa}$ which is within reasonable limits when compared to strength data observed from Kolsky bar experiments $[33,35]$ further validating our experimental technique. A reason for the increased strength lies within the partially higher yield strength scaling due to the pressure dependent shear modulus in the $\epsilon$ phase $\left(\left(1 / G_{0}\right)(\partial G / \partial P)=\right.$ $\left.0.0246 \mathrm{GPa}^{-1}\right)$ compared to the $\alpha$ phase $\left(\left(1 / G_{0}\right) \times\right.$ $\left.(\partial G / \partial P)=0.0221 \mathrm{GPa}^{-1}\right)$ [41]. The $\epsilon$ phase also exists at higher pressures so the scaling would result in higher strength in the hcp phase. While bcc materials generally show higher strength at these strain rates than hcp materials $[42,43]$, the strength increase may be explained by the transformation strain required to change the crystal structure from bcc to hcp $[12,43]$ and also the microstructural evolution during this phase transition. At approximately $13 \mathrm{GPa}$ of pressure, iron does not entirely transform into its $\epsilon$ phase but rather a mixed phase region is said to exist between 13-22 GPa [14,33]. According to recent experimental observations [44] and molecular dynamics simulations [45], the evolution of the $\epsilon$ phase begins at grain boundaries and these hcp clusters evolve into needlelike colonies which act as subgrains within the original grains. Shock experiments on single crystal iron [46] also indicate the formation of these nanocrystalline structures based on the symmetry-related variants of the new hcp phase from the parent bcc phase. In addition to these nanocrystals, twins form within the material to ensure kinematic compatibility between the two phases [47], but also due to the longitudinal wave during phase transformation [48] and the shear loading [35]. Given our loading conditions, it is possible that deformation twinning due to the transverse wave persists within our sample since the hcp phase favors twinning over slip as a deformation mechanism. Ultimately, the formation of hcp clusters and twins tends to occur during the transition in addition to the nucleation of screw dislocations, dislocation loops, stacking faults, and vacancies [45]. These twin boundaries and nanocrystalline grain boundaries, through the dynamic Hall-Petch effect, act as obstacles for dislocation motion therefore increasing dislocation pileup and inherently increasing the yield strength.

The strength of $\epsilon$ iron, at $10 \%$ shear strain, determined in the current study differs significantly from the previously reported values, Fig. 3(b). Ping et al. [19] have indirectly determined the yield strength of $\epsilon$ iron up to pressures of $560 \mathrm{GPa}$ from temperature measurements via laser shock experiments coupled with EXAFS data. The authors extrapolate their data and claim a yield strength of $\sim 20 \mathrm{GPa}$ and $\sim 30 \mathrm{GPa}$ at pressures of $50 \mathrm{GPa}$ and $100 \mathrm{GPa}$ respectively. Huntington et al. [20] arrived at a similar conclusion as Ping et al. and observed a flow strength of $\sim 40 \mathrm{GPa}$ at $100 \mathrm{GPa}$ of pressure by observing the growth of Rayleigh-Taylor instabilities. These strength reportings seem rather unexpected as they are on the cusp of the theoretical yield limit assuming the limit is a function of the pressure dependent shear modulus and given by $Y \propto G(P, T) / 2 \pi$. By extrapolating our data to higher pressures we claim these reportings to be an order of magnitude higher than the 4.3 and $5.1 \mathrm{GPa}$ yield strength we observe at pressures of $50 \mathrm{GPa}$ and $100 \mathrm{GPa}$, respectively. While this variability can be attributed to different grain sizes, strain rates, and heat treatment, these are not sufficient to explain a discrepancy of this magnitude. For example, recent MD simulations on nanocrystalline iron at strain rates of $10^{9} \mathrm{~s}^{-1}$ show a shear stress of $2.5 \mathrm{GPa}$ (yield stress of $4.3 \mathrm{GPa}$ ) at $30 \mathrm{GPa}$ of pressure [45]. Additionally, Prime et al. [49] recently conducted high strain-rate Richtmyer-Meshkov instability (RMI) experiments on 
tantalum and compared their results with Z-machine data [50]. The authors concluded pressure effects on strength are more significant than strain-rate effects in these extreme conditions. Hence, the main difference occurs due to experimental technique and the interpretation of the measurements. PSPI experiments are designed to probe the stress versus strain behavior and hence the strength of a material whereas the laser shock experiments by Ping et al. [19] were designed to understand the density, and temperature of compressed iron. This indirect determination of strength from temperature explains the high strength, on the order of theoretical yield strength $(G / 2 \pi)$, and large uncertainties in their results. Smith et al. [2] note the strength data from static experiments $[8,17]$ in pressure regimes below $300 \mathrm{GPa}$ were required to match the $300 \mathrm{~K}$ isotherm when converting their $\sigma_{x x}-\rho$ data to an isentropic $P-\rho$ curve. The reported strength from static experiments is on the same order as our results. However, our data does indicate an increased strength compared to Gleason and Mao [8] because of strain-rate dependence of $\epsilon$ iron. Diamond anvil cell experiments are typically conducted at compressive strain rates of $\sim 10^{-5} \mathrm{~s}^{-1}$ which is far lower than our experiments conducted at compressive strain rates of $\sim 10^{5} \mathrm{~s}^{-1}$.

In summary, PSPI experiments were conducted to characterize the pressure dependent plasticity and strength behavior of iron in its high pressure hep $\epsilon$ phase. From free surface velocity measurements coupled with modeling, we extract the complete stress-strain curve for $\epsilon$ iron for the first time and observe a substantial increase in the yield strength of the phase transformed material compared to its ambient $\alpha$ phase. Additionally, we find the dynamic strength of $\epsilon$ iron to be an order of magnitude lower than previous literature. Current work relies on static literature data for shear modulus scaling with pressure, however, future experiments are planned to dynamically characterize pressure dependent material properties for accurate construction of EOS and flow strength scaling. These strength and material parameters will be used to develop a sophisticated kinetics-based model for the phase transformation of iron. Our experiments on $\epsilon$ iron offer the potential to significantly extend our knowledge of the deformation behavior of Earth's inner core and construct a realistic model of the inner cores of rocky planets and meteorite impacts.

The authors gratefully acknowledge the support of DOE/ NNSA Award No. DE-NA0003957.

*vgandhi@caltech.edu

[1] W. B. Hubbard, W. J. Nellis, A. C. Mitchell, N. C. Holmes, S. S. Limaye, and P.C. McCandless, Science 253, 648 (1991).

[2] R. F. Smith et al., Nat. Astron. 2, 452 (2018).
[3] A. V. Jain and M. E. Lipschutz, Nature (London) 220, 139 (1968).

[4] K. Terada, T. Morota, and M. Kato, Nat. Commun. 11, 3453 (2020).

[5] A. Cameron, Icarus 126, 126 (1997).

[6] N. Hosono, S.-I. Karato, J. Makino, and T. R. Saitoh, Nat. Geosci. 12, 418 (2019).

[7] D. Batani et al., Phys. Rev. Lett. 88, 235502 (2002).

[8] A.E. Gleason and W. L. Mao, Nat. Geosci. 6, 571 (2013).

[9] D. Bancroft, E. L. Peterson, and S. Minshall, J. Appl. Phys. 27, 291 (1956).

[10] J. C. Jamieson and A. W. Lawson, J. Appl. Phys. 33, 776 (1962).

[11] D. H. Kalantar et al., Phys. Rev. Lett. 95, 075502 (2005).

[12] G. Krauss, Mater. Sci. Eng. 273-275, 40 (1999).

[13] S. Harjo, N. Tsuchida, J. Abe, and W. Gong, Sci. Rep. 7, 15149 (2017).

[14] J. C. Boettger and D. C. Wallace, Phys. Rev. B 55, 2840 (1997).

[15] B. Yaakobi, T. R. Boehly, D. D. Meyerhofer, T. J. B. Collins, B. A. Remington, P. G. Allen, S. M. Pollaine, H. E. Lorenzana, and J. H. Eggert, Phys. Rev. Lett. 95, 075501 (2005)

[16] K. J. Caspersen, A. Lew, M. Ortiz, and E. A. Carter, Phys. Rev. Lett. 93, 115501 (2004).

[17] R. J. Hemley, H. K. Mao, G. Shen, J. Badro, M. Hanfland, and D. Hausermann, Science 276, 1242 (1997).

[18] A. Singh, A. Jain, H. Liermann, and S. Saxena, J. Phys. Chem. Solids 67, 2197 (2006).

[19] Y. Ping et al., Phys. Rev. Lett. 111, 065501 (2013).

[20] C. M. Huntington et al., AIP Conf. Proc. 1793, 110007 (2017).

[21] C. Kettenbeil, Z. Lovinger, S. Ravindran, M. Mello, and G. Ravichandran, J. Dyn. Behav. Mater. 6, 489 (2020).

[22] R. Clifton and R. Klopp, Pressure Shear Plate Impact Testing (Metals Handbook, Metals Park, 1985), pp. 230239.

[23] C. Kettenbeil, M. Mello, M. Bischann, and G. Ravichandran, J. Appl. Phys. 123, 125902 (2018).

[24] O. T. Strand, D. R. Goosman, C. Martinez, T. L. Whitworth, and W. W. Kuhlow, Rev. Sci. Instrum. 77, 083108 (2006).

[25] See Supplemental Material at http://link.aps.org/ supplemental/10.1103/PhysRevLett.128.015705 for additional details regarding the experimental setup and data analysis, specimen preparation, velocity interferometry results, and additional details on EOS modeling, which also includes Refs. [26-30].

[26] J. A. Hawreliak et al., Phys. Rev. B 83, 144114 (2011).

[27] C. Kettenbeil, Dynamic strength of silica glasses at high pressures and strain rates, $\mathrm{Ph} . \mathrm{D}$. thesis, California Institute of Technology, 2019.

[28] S. Grunschel, Pressure-shear plate impact experiments on high-purity aluminum at temperatures approaching melt, Ph.D. thesis, Brown University, 2009.

[29] D. Dolan, Rev. Sci. Instrum. 81, 053905 (2010).

[30] M. B. Rauls and G. Ravichandran, J. Appl. Phys. 127, 065902 (2020). 
[31] Hibbitt, Karlsson, and Sorenson, ABAQUS/Explicit: User's Manual, Version 6.2 (Hibbitt, Karlsson and Sorenson Inc., United States, 2001).

[32] S. Ravindran, V. Gandhi, Z. Lovinger, M. Mello, and G. Ravichandran, Dyn. Behav. Mater. 7, 248 (2021).

[33] A. Sadjadpour, D. Rittel, G. Ravichandran, and K. Bhattacharya, Mech. Mater. 80, 255 (2015).

[34] D. J. Steinberg, S. G. Cochran, and M. W. Guinan, J. Appl. Phys. 51, 1498 (1980).

[35] D. Rittel, G. Ravichandran, and A. Venkert, Mater. Sci. Eng. 432, 191 (2006).

[36] D. B. Hayes, J. Appl. Phys. 46, 3438 (1975).

[37] D. Andrews, J. Comput. Phys. 7, 310 (1971).

[38] J. L. Brown, M. B. Prime, N. R. Barton, D. J. Luscher, L. Burakovsky, and D. Orlikowski, J. Appl. Phys. 128, 045901 (2020).

[39] J. Jung, Philos. Mag. A 43, 1057 (1981).

[40] H.-K. Mao, J. Shu, G. Shen, R. J. Hemley, B. Li, and A. K. Singh, Nature (London) 396, 741 (1998).

[41] M. Guinan and D. Steinberg, J. Phys. Chem. Solids 35, 1501 (1974).

[42] R. W. Armstrong and S. M. Walley, Int. Mater. Rev. 53, 105 (2008).
[43] G. T. (Rusty) Gray, Annu. Rev. Mater. Res. 42, 285 (2012).

[44] S. J. Wang, M. L. Sui, Y. T. Chen, Q. H. Lu, E. Ma, X. Y. Pei, Q.Z. Li, and H. B. Hu, Sci. Rep. 3, 1086 (2013).

[45] H.-T. Luu, R. J. Ravelo, M. Rudolph, E. M. Bringa, T. C. Germann, D. Rafaja, and N. Gunkelmann, Phys. Rev. B 102, 020102(R) (2020).

[46] J. A. Hawreliak, D. H. Kalantar, J. S. Stölken, B. A. Remington, H. E. Lorenzana, and J. S. Wark, Phys. Rev. B 78, 220101(R) (2008).

[47] K. Bhattacharya, Microstructure of Martensite: Why It Forms and How It Gives Rise to the Shape-Memory Effect? (Oxford University Press, New York, 2012).

[48] N. Gunkelmann, D. R. Tramontina, E. M. Bringa, and H. M. Urbassek, J. Appl. Phys. 117, 085901 (2015).

[49] M. B. Prime, W. T. Buttler, S. J. Fensin, D. R. Jones, J. L. Brown, R. S. King, R. Manzanares, D. T. Martinez, J. I. Martinez, J. R. Payton, and D. W. Schmidt, Phys. Rev. E 100, 053002 (2019).

[50] J. L. Brown, C. S. Alexander, J. R. Asay, T. J. Vogler, D. H. Dolan, and J. L. Belof, J. Appl. Phys. 115, 043530 (2014). 